\title{
Polaron relaxation in a quantum dot due to anharmonic coupling within a mean-field approach
}

\author{
Tobias Stauber* and Mikhail I. Vasilevskiy \\ Centro de Física, Universidade do Minho, Campus de Gualtar, 4710-057 Braga, Portugal
}

(Received 13 February 2009; published 25 March 2009)

\begin{abstract}
We study the electronic relaxation in a quantum dot within the polaron approach by focusing on the reversible anharmonic decay of longitudinal optical (LO) phonons forming the polaron into longitudinalacoustic (LA) phonons. The coherent coupling between the LO and LA phonons is treated within a mean-field approach. We derive a temperature-dependent interlevel coupling parameter, related to the Grüneisen parameter and the thermal-expansion coefficient, which characterizes an effective decay channel for the electronic (or excitonic) states. Within this theory, we obtain a characteristic anharmonic decay time of $1 \mathrm{~ns}, 2-3$ orders of magnitude longer than previous predictions based on the Fermi's Golden Rule. We suggest that coherent relaxation due to carrier-carrier interaction is an efficient alternative to the (too slow) polaron decay.
\end{abstract}

DOI: 10.1103/PhysRevB.79.113301

PACS number(s): 78.67.Hc, 71.38.-k, 73.61.Ey, 73.61.Ga

\section{INTRODUCTION}

The understanding of the relaxation mechanisms in quantum dots (QDs) is important from both the fundamental and technological points of view since QDs are increasingly finding applications in lasers, single-photon emitters, and possibly quantum computers where fast carrier dynamics are indispensable. Whereas in bulk semiconductors, the hot carrier relaxation is mediated via emission of optical phonons, ${ }^{1}$ in a QD the discrete electron and hole levels are separated by energies that generally do not match any phonon energy. This simple idea gave rise to the "phonon bottleneck" concept which predicts the inefficiency of hot carrier relaxation by emission of phonons in QDs. ${ }^{2}$ However, this prediction relies on the assumption that the phonon emission is irreversible, with a probability described by the Fermi's Golden Rule (FGR).

It has then been realized that multiple-scattering processes are important and that the electron-phonon (e-ph) interaction in QDs must be treated in a nonperturbative ${ }^{3}$ and nonadiabatic ${ }^{4}$ way, leading to the energy spectra described by polaronic quasiparticle excitations. Polaron spectra of QDs have been considered theoretically using several approaches, ${ }^{5-7}$ and various steady-state observable properties [such as photoluminescence (PL) and Raman spectra] have been calculated and compared to experimental data, convincingly demonstrating the importance of the polaron concept. ${ }^{3,4,7-10}$

One might then question whether the phonon bottleneck in QDs really exists and, in fact, several experiments point in either direction. On one hand, an efficient relaxation of optically created electron-hole pairs (hereafter called excitons) was reported in a number of works studying self-assembled QDs (SAQDs), ${ }^{11-13}$ with both PL rise time ${ }^{11,12}$ and photoinduced intraband absorption decay time ${ }^{13}$ below or of the order of 10 ps. Recent studies ${ }^{14,15}$ performed on chemically grown nanocrystals (NCs), where exciton energy-level spacings are larger than in SAQDs, also revealed ultrafast intraband relaxation although the mechanism seems to be different for $\mathrm{CdSe}$ and PbSe NCs. There are some works where the relaxation of a lone carrier (e.g., electron in an $n$-doped QD) was studied. ${ }^{9,16,17}$ Their results suggest that the relaxation is slower than for excitons but still fast, with a characteristic time of several tens of picoseconds for $n$-doped InAs/GaAs SAQDs. ${ }^{9,16}$ On the other hand, there are published experimental results that support the existence of a phonon bottleneck effect in the relaxation of optically created excitons, both in self-assembled ${ }^{18,19}$ and nanocrystal ${ }^{20,21}$ QDs. For instance, a relaxation time of $7.7 \mathrm{~ns}, \sim 15$ times the radiation lifetime, was obtained for InAs/GaAs SAQDs in Ref. 18.

The polaron model can explain intraband relaxation of carriers in QDs only within the context of so-called pseudorelaxation $^{22}$ (i.e., oscillatory dynamics) since polarons are stationary states of an electron (exciton) coupled to optical phonons. Some additional interactions should therefore be responsible for the true polaron relaxation (i.e., thermalization). Several possible mechanisms of hot carrier relaxation in QDs have been proposed:

(i) The polaron has a rather short lifetime ${ }^{23}$ because of the anharmonic effects that lead to a fast decay of confined optical phonons forming the polaron. This mechanism has been considered for both exciton ${ }^{24}$ and lone electron ${ }^{25,26}$ relaxations.

(ii) In SAQDs, the polaron can relax via an Auger-type mechanism assisted by electrons present in the wetting layer where the energy spectrum is continuous. ${ }^{27,28}$

(iii) Acoustic phonons can provide the possibility of transitions between different (exciton-) polaron states. ${ }^{7}$ If the acoustic phonon spectrum is (at least partially) continuous and the polaron spectrum is sufficiently dense, this interaction would drive the polaron dynamics toward equilibrium.

An Auger-type mechanism was also proposed ${ }^{29,30}$ for the relaxation in chemically grown QDs, according to which the excess energy is first transferred from the electron to the QD hole through their Coulomb interaction, and the subsequent hole cooling occurs via emission of acoustic phonons because the hole level spacings are relatively small and match the continuum of acoustic phonon energies. Experimental data support this mechanism, at least for $\mathrm{CdSe}$ nanocrystals. ${ }^{14,15,31}$ If one uses the exciton-polaron language, this mechanism is equivalent to (iii).

In this Brief Report, we show that the anharmonicity mechanism (i) is too slow and therefore not relevant in most of the experimental situations. It was initially proposed and illustrated for the simple case of a single longitudinal optical 
(LO) phonon mode coupled to two electron states in Ref. 23 using the rotating-wave approximation. Later, more realistic calculations of the polaron spectrum and relaxation rate were performed for an exciton ${ }^{24}$ and a lone electron ${ }^{25,26}$ in an InAs/GaAs SAQD. In the latter work, ${ }^{26}$ a good agreement with experimental findings of Ref. 16 has been achieved by including several anharmonic phonon decay channels known in the literature for various materials. ${ }^{32}$ However, in all these works the LO phonon decay was considered to be irreversible and FGR was used to calculate the polaron relaxation rate. We shall avoid this approximation, which is known to be a rather crude one for QDs and incoherent with the polaron concept. Instead, we develop a nonperturbative meanfield theory of the QD polaron coupled to acoustic phonons through reversible anharmonic processes, resulting in a much slower relaxation. Our main conclusion is that the mechanism (i) is irrelevant in most experimental situations.

\section{MODEL AND APPROACH}

We present a nonperturbative approach to the nonequilibrium problem of electron relaxation in a quantum dot. This shall be discussed within a simple (minimal) model which nevertheless includes all relevant interaction terms. Our QD contains two nondegenerate electronic levels (separated by an energy $\epsilon$ ), coupled to a single confined LO phonon mode. The LO phonons can reversibly decay into a couple of longitudinal-acoustic (LA) phonons through an anharmonic process that has been identified experimentally for some bulk semiconductors. ${ }^{32}$ We shall also include the possibility of further anharmonic interactions of these (secondary) LA phonons with other vibrational modes in the QD or its surroundings. The Hamiltonian of this model can be written as follows:

$$
H=H_{\mathrm{pol}}+H_{\mathrm{LA}}+H_{\text {int }}+H_{\text {anharm }},
$$

where the polaron Hamiltonian is given by

$$
H_{\mathrm{pol}}=\epsilon c_{1}^{\dagger} c_{1}+\omega_{B} b^{\dagger} b+M\left(c_{1}^{\dagger} c_{0}+c_{0}^{\dagger} c_{1}\right)\left(b+b^{\dagger}\right),
$$

the Hamiltonian of the acoustic phonons reads

$$
H_{\mathrm{LA}}=\omega_{A} a^{\dagger} a,
$$

and the interaction term is

$$
H_{\text {int }}=G\left(a^{\dagger} a^{\dagger} b+b^{\dagger} a a\right) \text {. }
$$

In Eqs. (2) and (3), $\omega_{B}$ and $\omega_{A}$ are the energies of the $\mathrm{LO}$ and LA phonons, respectively (we set $\hbar=1$ ), and $M$ is the interlevel $e$-ph coupling constant. ${ }^{33}$ In Eq. (4), $G$ denotes the characteristic energy of the $\mathrm{LO} \leftrightarrow 2 \mathrm{LA}$ process related to the experimentally measured anharmonic decay time constant. ${ }^{32,34}$ The last term in Eq. (1) stands for anharmonic interactions of the acoustic phonons (excluding those written explicitly as $\left.H_{\text {int }}\right)$. This term is needed for a well-defined ground state (see below).

In the spirit of a mean-field approach, the interaction term shall be approximated as

$$
H_{\text {int }}=G\left(\left\langle a^{\dagger} a^{\dagger}\right\rangle b+b^{\dagger}\langle a a\rangle+a^{\dagger} a^{\dagger}\langle b\rangle+\left\langle b^{\dagger}\right\rangle a a\right),
$$

thus neglecting the coupling of the fluctuations of the bosonic fields. By defining shifted bosonic modes, $b \rightarrow b$
$+G\left\langle a^{\dagger} a^{\dagger}\right\rangle / \omega_{B}$, Hamiltonian (1) can be written as

$$
H=H_{\mathrm{pol}}+\widetilde{H}_{\mathrm{LA}}+\Delta H-\frac{\left(G\left\langle a^{\dagger} a^{\dagger}\right\rangle\right)^{2}}{\omega_{B}},
$$

where we have

$$
\Delta H=-\tilde{M}\left(c_{1}^{\dagger} c_{0}+c_{0}^{\dagger} c_{1}\right),
$$

and

$$
\widetilde{H}_{\mathrm{LA}}=\omega_{A} a^{\dagger} a+D\left(a^{\dagger} a^{\dagger}+a a\right) .
$$

In Eq. (7), we have introduced an effective coupling parameter,

$$
\tilde{M}=M \frac{2 G\left\langle a^{\dagger} a^{\dagger}\right\rangle}{\omega_{B}},
$$

and in Eq. (8) an effective coupling constant $D$ including all the anharmonicity effects involving the LA phonons. This constant takes into account the effect of the last two terms in Eq. (5) as well as $H_{\text {anharm }}$ which represents fourth-order processes with the participation of two LA phonons, i.e., $D$ $=G\langle b\rangle+\tilde{D}$.

There is a new direct relaxation term $\Delta H$, characterizing the new relaxation scale $\tau=1 / \tilde{M}$. To quantify this scale, we need to know the value of the anomalous amplitude $\left\langle a^{\dagger} a^{\dagger}\right\rangle$. The explicit evaluation of the anomalous amplitude involves the diagonalization of $\widetilde{H}_{\mathrm{LA}}$, which can be made using a Bogoljubov-Valatin transformation, ${ }^{35} \eta=g a+h a^{\dagger}$, with the normalization of the canonical commutator relations $g^{2}-h^{2}$ $=1$. This gives

$$
g=\frac{D}{\widetilde{\omega}_{A}} \frac{1}{h}, \quad h=\sqrt{\frac{\omega_{A}-\widetilde{\omega}_{A}}{2 \widetilde{\omega}_{A}},}
$$

with the renormalized eigenfrequency

$$
\widetilde{\omega}_{A}=\sqrt{\omega_{A}^{2}-4 D^{2}} .
$$

The occupation number of the acoustic phonons can be expressed in terms of the expectation value of the new bosons,

$$
\left\langle\eta^{\dagger} \eta\right\rangle \equiv n_{\text {bos }}=\left(e^{\widetilde{\omega}_{A} /\left(k_{B} T\right)}-1\right)^{-1},
$$

as

$$
n_{A} \equiv\left\langle a^{\dagger} a\right\rangle=n_{\text {bos }}+h^{2}\left(1+2 n_{\text {bos }}\right) .
$$

The anomalous amplitude entering Eq. (9) is given by

$$
\langle a a\rangle=\left\langle a^{\dagger} a^{\dagger}\right\rangle=-g h\left(1+2 n_{\text {bos }}\right) .
$$

Thus, all interaction terms in Eq. (6) (except $H_{\text {pol }}$ ) are now expressed in terms of the effective coupling constant $D$. In particular, the interlevel coupling constant (9) is given by

$$
\tilde{M}=-M \frac{2 G D}{\widetilde{\omega}_{A} \omega_{B}}\left(1+2 n_{\text {bos }}\right) .
$$

\section{Self-consistent mean-field theory}

In a self-consistent mean-field theory involving only electrons and LO and LA phonons, we should put $D=G\langle b\rangle$. The 
self-consistency condition is then defined by the following two equations:

$$
\begin{gathered}
\langle b\rangle=-\frac{2 G\langle a a\rangle}{\omega_{B}}, \\
\langle a a\rangle=\frac{-G\langle b\rangle}{\sqrt{\omega_{A}^{2}-4(G\langle b\rangle)^{2}}}\left(1+2 n_{\mathrm{bos}}\right) .
\end{gathered}
$$

This yields the following self-consistent equation for the anomalous amplitude:

$$
\omega_{A}^{2} \omega_{B}^{2}-16 G^{4}\langle a a\rangle^{2}=4 G^{4}\left(1+2 n_{\text {bos }}\right)^{2} .
$$

For $G \rightarrow 0$, we thus have

$$
\langle a a\rangle \approx \omega_{A} \omega_{B} /\left(4 G^{2}\right) ; \quad \tilde{M}_{\mathrm{MF}} \approx M \omega_{A} /(2 G) .
$$

This means that the characteristic decay time, $\tau \sim 1 / \tilde{M}_{\mathrm{MF}}$, tends to zero for vanishing anharmonic coupling, which certainly is an artifact of the model. The physical reason for this is the omission of other anharmonic processes $(\tilde{D}=0)$. It is well known that self-consistent mean-field theories for boson-fermion systems may lead to such inconsistencies. ${ }^{36}$

\section{EFFECTIVE ANHARMONIC COUPLING CONSTANT}

In order to overcome the difficulty of the previous section, we have to take into account the fact that $\mathrm{LO} \leftrightarrow 2 \mathrm{LA}$ is not the only anharmonic process involving the LA phonons considered in our model. In Eq. (8) we have introduced $D$ as an effective coupling constant representing all the third- and fourth-order processes with the participation of two LA phonons. It will now be considered independent of $G$, which is the coupling constant of one particular anharmonic process. Assuming that we can ignore other cubic anharmonicity effects (involving just one LA phonon), we can relate $D$ to the experimentally known Grüneisen parameter, ${ }^{37} \gamma_{G r}$ $=-d \ln \theta / d \ln V$, where $\theta$ is the Debye temperature and $V$ is the crystal volume. In the Grüneisen approximation, the variation in all vibrational frequencies with the crystal volume is assumed to be the same, ${ }^{37} d \ln \theta=d \ln \omega$. If we consider the effective coupling constant as a function of temperature, $D(T)$, and use Eq. (11) for $\widetilde{\omega}_{A}$, then we can write:

$$
\gamma_{\mathrm{Gr}}=-\frac{d \ln \widetilde{\omega}_{A}}{d \ln V}=-\frac{1}{\alpha_{T}} \frac{d \ln \widetilde{\omega}_{A}}{d T}=-\frac{1}{\alpha_{T}}\left(-\frac{2}{\widetilde{\omega}_{A}^{2}} \frac{d D^{2}}{d T}\right),
$$

where $\alpha_{T}$ is the thermal-expansion coefficient. Thus, we obtain the following equation for $D(T)$,

$$
\frac{2}{\omega_{A}^{2}-4 D^{2}} \frac{d D^{2}}{d T}=\gamma_{\mathrm{Gr}} \alpha_{T}
$$

Its solution, with the condition $D(0)=0$, yields

$$
D(T)=\frac{\omega_{A}}{2}\left[1-\exp \left(-2 \gamma_{\mathrm{Gr}} \alpha_{T} T\right)\right]^{1 / 2} .
$$

With typical values of $\alpha_{T} \approx 10^{-5} \mathrm{~K}^{-1}$ and $\gamma_{\mathrm{Gr}} \approx 1.5$, we obtain $D / \omega_{A} \approx 1 / 20$ for $T \approx 300 \mathrm{~K}$. Accordingly, $\widetilde{\omega}_{A} \approx \omega_{A}$. Fig-

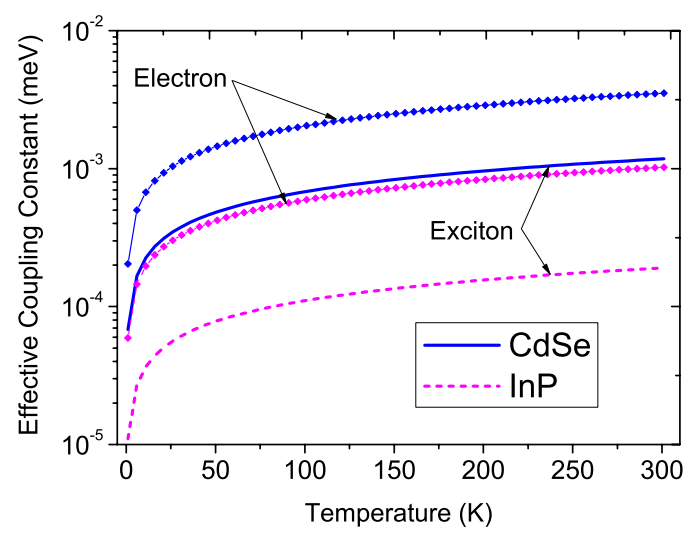

FIG. 1. (Color online) Effective anharmonic interlevel coupling parameter $\tilde{M}$ calculated for excitons and lone electrons in CdSe and InP nanocrystal QDs of $2 \mathrm{~nm}$ in radius.

ure 1 shows the temperature dependence of the effective interlevel coupling parameter $\tilde{M}$ following from Eqs. (13) and (18), calculated for spherical QDs. We assumed $\omega_{A}=\omega_{B} / 2$, and used the anharmonic decay constants from experiment $\left[G=0.15 \mathrm{ps}^{-1}\right.$ for CdSe (Ref. 34) and $0.04 \mathrm{ps}^{-1}$ for InP (Ref. 32)] and calculated $e$-ph coupling parameters. ${ }^{8,38} \tilde{M}$ thus turns out to be very small (even for electron in $\mathrm{CdSe}$ QD), much smaller than the typical $e$-ph interaction energy ( $\approx 3 \mathrm{meV}$ for excitons and $\approx 20 \mathrm{meV}$ for electrons interacting with spherical-symmetric phonons). It is larger for lone electron, compared to exciton, because of the stronger coupling to polar phonons. ${ }^{39}$

To conclude, even though the anharmonic decay of LO phonons is faster in QDs than in bulk semiconductors because of the absence of the restrictions due to momentum conservation, our results indicate that the energy scale of this interaction is far below the other relevant characteristic energies. Consequently, this effective decay channel is too slow to account for the experimental data. On the contrary, coherent coupling between carriers can produce effective (pseudo)relaxation of the QD polaron, with $\tau \sim 10 \mathrm{ps,} \mathrm{even} \mathrm{if}$ there are no dissipative relaxation channels present. ${ }^{22}$

\section{SUMMARY}

Assuming the coherent coupling between the optical and acoustic phonons and using a mean-field approximation, Hamiltonian (1) describing the interacting polaron can be reduced to $H=H_{\text {pol }}+\Delta H$ with $\Delta H$ given by Eqs. (7), (13), and (18). Note that $\Delta H$ is temperature dependent, and [apart from the parameters of the "noninteracting" Hamiltonian (2) and (3)] entirely determined by the thermal-expansion coefficient and the Grüneisen parameter. The term $\Delta H$ corresponds to a (pseudo)relaxation time $\tau \sim 1 \mathrm{~ns}$, i.e., $2-3$ orders of magnitude larger than the previous predictions based on the Fermi's Golden rule. This renders the anharmonic decay channel virtually irrelevant, at least for nanocrystal QDs where the polaron is formed by a small number of confined optical phonon modes most strongly coupled to the electron or exciton. Instead, the temporal evolution of the photoin- 
duced polarization in a QD can proceed much faster just because of the quantum beats resulting from coherent manybody interactions. ${ }^{22}$ In doped SAQD heterostructures, the relaxation of the lone electron or hole occurs due to the coupling to the carriers in the wetting layer ${ }^{28}$ while in optically excited QDs the same mechanism operates because of the electron-hole interaction. ${ }^{30}$ In some cases, this pseudorelaxation can be taken over by the true (dissipative) one mediated by the continuum of acoustic phonons. ${ }^{7}$ In fact, atomistic calculations using the time-domain $a b$ initio approach indicate that the relaxation in nanocrystals occurs via $e$-ph coupling to acoustic-type vibrations and the phonon bottle- neck can exist only at low energies in very small dots. ${ }^{40} \mathrm{We}$ finally note that recent experiments ${ }^{41}$ have shown a bottleneck effect on the hole relaxation in CdSe QDs of 1-3 nm in radius (with the gap between the ground and first-excited states $\approx 30-100 \mathrm{meV}$, similar to SAQDs), which is consistent with the conclusions of this work.

\section{ACKNOWLEDGMENT}

Support from the FCT (Portugal) through Projects No. PTDC/FIS/64404/2006 and No. PTDC/FIS/72843/2006 is acknowledged. *tobias.stauber@fisica.uminho.pt

${ }^{1}$ C. Fürst, A. Leitenstorfer, A. Laubereau, and R. Zimmermann, Phys. Rev. Lett. 78, 3733 (1997).

${ }^{2}$ U. Bockelmann and G. Bastard, Phys. Rev. B 42, 8947 (1990).

${ }^{3}$ S. Hameau, Y. Guldner, O. Verzelen, R. Ferreira, G. Bastard, J. Zeman, A. Lemaitre, and J. M. Gerard, Phys. Rev. Lett. 83, 4152 (1999).

${ }^{4}$ V. M. Fomin, V. N. Gladilin, J. T. Devreese, E. P. Pokatilov, S. N. Balaban, and S. N. Klimin, Phys. Rev. B 57, 2415 (1998).

${ }^{5}$ T. Stauber, R. Zimmermann, and H. Castella, Phys. Rev. B 62 , 7336 (2000).

${ }^{6}$ L. Jacak, P. Machnikowski, J. Krasnyj, and R. Zöller, Eur. Phys. J. D 22, 319 (2003).

${ }^{7}$ M. I. Vasilevskiy, E. V. Anda, and S. S. Makler, Phys. Rev. B 70, 035318 (2004).

${ }^{8}$ R. P. Miranda, M. I. Vasilevskiy, and C. Trallero-Giner, Phys. Rev. B 74, 115317 (2006).

${ }^{9}$ S. Sauvage, P. Boucaud, R. P. S. M. Lobo, F. Bras, G. Fishman, R. Prazeres, F. Glotin, J. M. Ortega, and J. M. Gerard, Phys. Rev. Lett. 88, 177402 (2002)

${ }^{10}$ D. Sarkar, H. P. van der Meulen, J. M. Calleja, J. M. Becker, R. J. Haug, and K. Pierz, Phys. Rev. B 71, 081302(R) (2005).

${ }^{11}$ S. Marcinkevičius, A. Gaarder, and R. Leon, Phys. Rev. B 64, 115307 (2001).

${ }^{12}$ K. W. Sun, A. Kechiantz, B. C. Lee, and C. P. Lee, Appl. Phys. Lett. 88, 163117 (2006).

${ }^{13}$ T. Müller, F. F. Schrey, G. Strasser, and K. Unterrainer, Appl. Phys. Lett. 83, 3572 (2003).

${ }^{14}$ R. D. Schaller, J. M. Pietryga, S. V. Goupalov, M. A. Petruska, S. A. Ivanov, and V. I. Klimov, Phys. Rev. Lett. 95, 196401 (2005).

${ }^{15}$ E. Hendry, M. Koeberg, F. Wang, H. Zhang, C. de Mello Donegá, D. Vanmaekelbergh, and M. Bonn, Phys. Rev. Lett. 96, 057408 (2006).

${ }^{16}$ E. A. Zibik et al., Phys. Rev. B 70, 161305(R) (2004).

${ }^{17}$ P. Guyot-Sionnest, B. Wehrenberg, and D. Yu, J. Chem. Phys. 123, 074709 (2005).

${ }^{18}$ R. Heitz, H. Born, F. Guffarth, O. Stier, A. Schliwa, A. Hoffmann, and D. Bimberg, Phys. Rev. B 64, 241305(R) (2001).

${ }^{19}$ J. Urayama, T. B. Norris, J. Singh, and P. Bhattacharya, Phys. Rev. Lett. 86, 4930 (2001).

${ }^{20}$ P. Guyot-Sionnest, M. Shim, C. Matranga, and M. Hines, Phys. Rev. B 60, R2181 (1999).
${ }^{21}$ A. J. Nozik, Annu. Rev. Phys. Chem. 52, 193 (2001).

${ }^{22}$ T. Stauber and R. Zimmermann, Phys. Rev. B 73, 115303 (2006).

${ }^{23}$ X. Q. Li, H. Nakayama, and Y. Arakawa, Phys. Rev. B 59, 5069 (1999).

${ }^{24}$ O. Verzelen, R. Ferreira, and G. Bastard, Phys. Rev. Lett. 88, 146803 (2002)

${ }^{25}$ O. Verzelen, R. Ferreira, and G. Bastard, Phys. Rev. B 62, R4809 (2000).

${ }^{26} \mathrm{~T}$. Grange, R. Ferreira, and G. Bastard, Phys. Rev. B 76, 241304(R) (2007).

${ }^{27}$ Y. Toda, O. Moriwaki, M. Nishioka, and Y. Arakawa, Phys. Rev. Lett. 82, 4114 (1999)

${ }^{28}$ J. Seebeck, T. R. Nielsen, P. Gartner, and F. Jahnke, Phys. Rev. B 71, 125327 (2005).

${ }^{29}$ Al. L. Efros, V. A. Kharchenko, and M. Rosen, Solid State Commun. 93, 281 (1995).

${ }^{30}$ G. A. Narvaez, G. Bester, and A. Zunger, Phys. Rev. B 74, 075403 (2006)

${ }^{31}$ R. R. Cooney, S. L. Sewall, K. E. H. Anderson, E. A. Dias, and P. Kambhampati, Phys. Rev. Lett. 98, 177403 (2007).

${ }^{32}$ F. Vallée, Phys. Rev. B 49, 2460 (1994); S. Barman and G. P. Srivastava, ibid. 69, 235208 (2004).

${ }^{33}$ Note that we omitted the intralevel coupling terms for simplicity which can be included within a rotating-wave approximation, see Ref. 5.

${ }^{34}$ S. S. Prabhu, A. S. Vengurlekar, S. K. Roy, and J. Shah, Phys. Rev. B 51, 14233 (1995).

${ }^{35}$ G. D. Mahan, Many-Particle Physics (Kluwer Academic, New York, 2000).

${ }^{36}$ J. Ranninger and S. Robaszkiewicz, Physica B \& C 135, 468 (1985).

${ }^{37}$ M. Born and K. Huang, Dynamical Theory of Crystal Lattices (Clarendon, Oxford, 1996).

${ }^{38}$ M. Hamma, R. P. Miranda, M. I. Vasilevskiy, and I. Zorkani, J. Phys.: Condens. Matter 19, 346215 (2007).

${ }^{39}$ Note that this conclusion is in contradiction with the experimental data (see Sec. I) and therefore supports the doubts concerning the efficiency of the anharmonic decay mechanism.

${ }^{40}$ O. V. Prezhdo, Chem. Phys. Lett. 460, 1 (2008).

${ }^{41}$ S. Xu, A. A. Mikhailovsky, J. A. Hollingsworth, and V. I. Klimov, Phys. Rev. B 65, 045319 (2002). 